\title{
Zebrafish: Recomendações de Manutenção em Laboratório
}

\author{
Zebrafish: Laboratory Maintenance Recommendations
}

\author{
Carla Letícia Gediel Rivero-Wendt*a; Rosemary Matias ${ }^{a}$; Giovana Coutinho Zulin do Nascimento ${ }^{\text {; }}$ S Silvia Cristina \\ Heredia ${ }^{\mathrm{a}}$; Magno Rafael Miranda Santos ${ }^{\mathrm{b}}$; Osvaldo Borges Pinto Júnior ${ }^{\mathrm{c}}$
}
aUniversidade Anhanguera-Uniderp, Programa de Pós-Graduação Stricto Sensu em Meio Ambiente e Desenvolvimento Regional. MS, Brasil. bUniversidade de Cuiabá, Programa de Pós-Graduação Stricto Sensu em Ambiente e Saúde. MT, Brasil.
'Universidade de Cuiabá, Programa de Pós-Graduação Stricto Sensu em Ciências Ambientais. MT, Brasil. *E-mail: leticiagediel@gmail.com

\begin{abstract}
Resumo
O zebrafish ou paulistinha (Danio rerio) são cada vez mais utilizados em pesquisas científicas incluindo a pesquisa básica e aplicada nas áreas de biomedicina, toxicologia, ciências ambientais e aquicultura. No Brasil, os biotérios automatizados são escassos, têm custo oneroso e para fazer uso do modelo animal são necessárias adaptações para criação e manutenção da espécie. Deve existir a compreensão de que as adaptações devem respeitar os padrões de qualidade, implicando na diminuição de mortalidade e doenças nos aquários de manutenção. O objetivo desta revisão de literatura é apresentar as recomendações de protocolo de manutenção do zebrafish, em condições de laboratório, sem o uso de biotério automatizado. Apesar da gama de informações e diferentes métodos de manutenção encontrados para a espécie, fica claro que em condições de laboratório se deve manter o animal nas melhores condições, possibilitando qualidade no desenvolvimento e na reprodução para realizar experimentos com confiabilidade de execução.
\end{abstract}

Palavras-chave: Desenvolvimento. Ambiente e Sustentabilidade. Danio rerio. Biotério.

\begin{abstract}
The use of Zebrafish or paulistinha (Danio rerio) has increased considerably in basic and applied scientific research as biomedicine, toxicology, environmental sciences and aquaculture. In Brazil, automated experimental animal facilities are scarce and costly. To make use of this animal model, adaptations are required for creation and maintenance. These changes must respect quality standards, providing mortality and disease reduction in aquariums maintenance. The literature review aim is to synthesize recommendations for the zebrafish maintenance protocol in laboratory conditions without the automated experimental animal facility. Despite the information range and different maintenance methods found for the species, it is clear that under laboratory conditions, the animal must be kept in the best conditions, enabling development and reproduction quality to be used in experiments with reliable execution.
\end{abstract}

Keywords: Development. Environment and Sustainability. Danio rerio. Experimental Animal Facility.

\section{Introdução}

O Danio rerio (zebrafish) é uma espécie de água doce com pequeno porte. Possui $70 \%$ de homologia genética e fisiológica com os seres humanos, apresentando vantagens em seu uso para pesquisas em relação aos camundongos e ratos. Além disso, são modelos com alta taxa de reprodução, com rápido desenvolvimento e a manutenção em laboratório representa baixo custo.

O zebrafish provou ser adequado para triagem genética, em grande escala, seja em busca de modelos para doença humana ou para mecanismos de controle relacionados à diferenciação natural ou patológica de células e tecidos, quando comparados aos modelos de mamíferos, que são mais difíceis de realizar em função do longo período de gestação intrauterina (D'ANGELO et al., 2016).

Estudos internacionais têm considerado como parâmetros de cuidados e manutenção a habitação física, nutrição, patógenos e qualidade da água (LAWRENCE; EISEN; VARGA, 2016; LIDSTER et al., 2017). Existe uma tendência para a padronização dos protocolos relacionados ao zebrafish
(GEISLER et al., 2016; GOODWIN et al., 2016a, 2016b). Os biotérios automatizados oferecem um controle dos parâmetros de tratamento de água com filtros mecânicos, químico e biológico, monitoramento em tempo real da temperatura e condutividade, aquecimento ajustado entre 20 e $30^{\circ} \mathrm{C}$, troca automática da água do sistema, alarme sonoro e luminoso, em caso de filtro sujo ou obstruído, sistema de drenos que evitam transbordamento e acionamento diário de luz UV com ciclos de 1 a 12 horas (ALESCO, 2020).

Em função de todas estas especificidades protocolares, esta revisão tem como objetivo recomendar protocolos específicos de manutenção do zebrafish em condições laboratoriais.

\section{Desenvolvimento}

\subsection{Biologia da espécie}

O Danio rerio (zebrafish) pertence à família Cyprinidae, sendo um peixe tropical originário da Ásia, vivem em cardumes com ambos os sexos. Apesar de exercerem comportamento social, apresentam comportamento agonista quando acasalam e são territorialistas entre os sexos (LAWRENCE, 2007). 
O espécime adulto possui corpo esguio, alongado, cabeça curta, boca inclinada, a mandíbula superior possui uma protrusão que facilita a abertura da boca e sucção de alimentos (LAWRENCE, 2007). A característica marcante da espécie são as listras ao longo do corpo e nas nadadeiras anal e caudal (Figura 1).

Figura 1 - Adulto de Danio rerio (zebrafish) evidenciando as listras ao longo do corpo

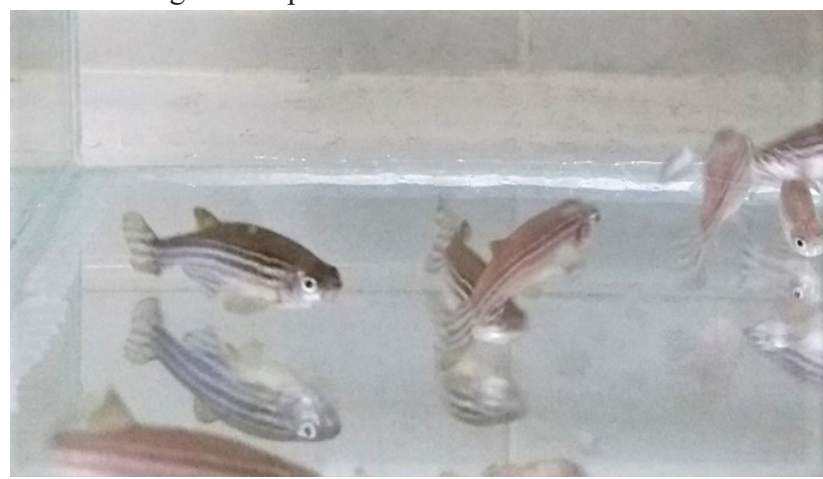

Fonte: Os autores.

No estágio embrionário larval e juvenil (Figura 2), o embrião já apresenta órgãos definidos, linha lateral (neuromastos), nada livremente, já evidencia bexiga natatória cheia e quando juvenil já apresenta as nadadeiras e o padrão de pigmentação dos adultos (LAWRENCE, 2007).

Figura 2 - Embriões de Danio rerio (zebrafish) com 48 horas após fertilização (a); Larva recém eclodida com 72 horas (b)

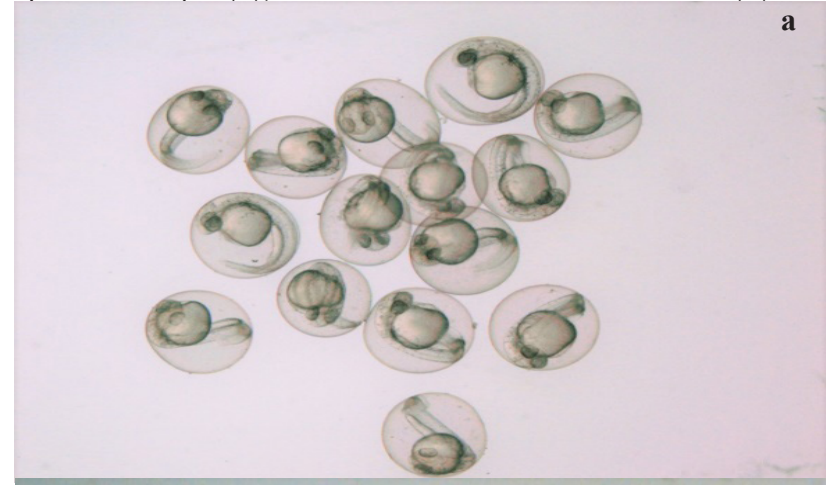

Fonte: Os autores

A reprodução da espécie é influenciada pelo fotoperíodo, a desova começa nas primeiras horas do dia. O zebrafish se reproduz, de forma contínua, na maturidade sexual. As fêmeas podem produzir ovos diariamente, entretanto, é recomendado um intervalo de pelo menos 15 dias entre os cruzamentos. A eficácia do processo de reprodução será verificada de acordo com o número de ovos viáveis por cruzamento (Figura 3).

Figura 3 - Ovos de Danio rerio (zebrafish) viável (a) e não viável (b)
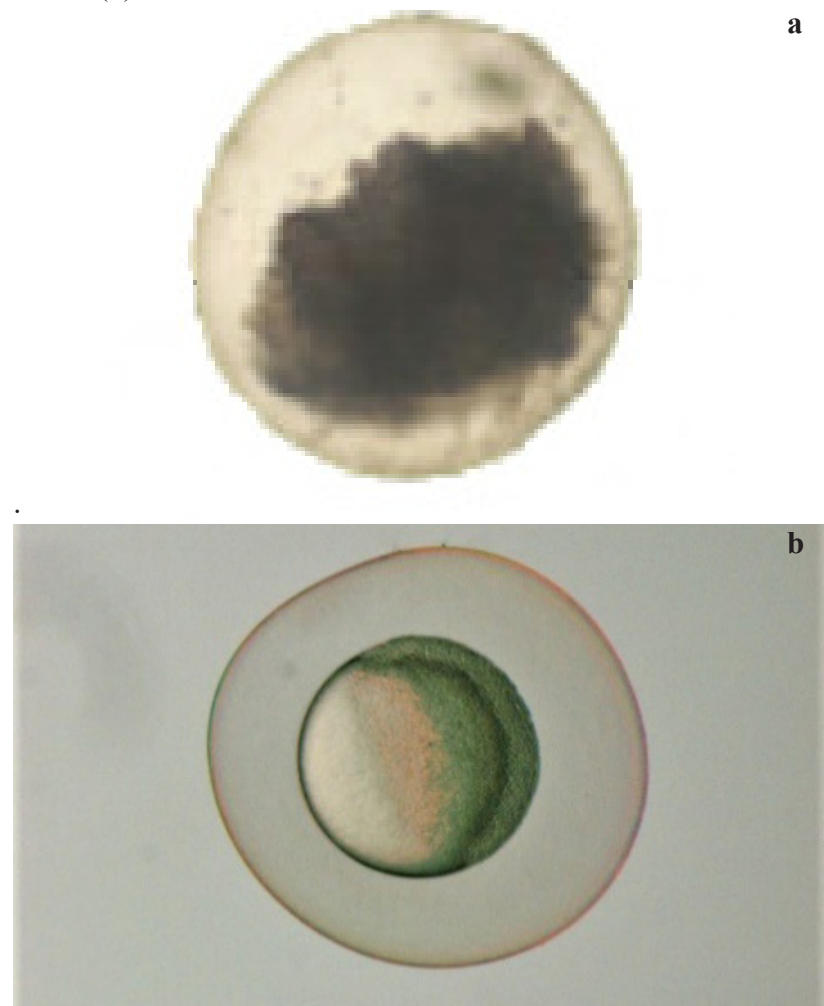

Fonte: Os autores.

A liberação dos ovos e espermatozoides ocorre, de forma simultânea, sendo a fecundação externa. O sistema preparado para o acasalamento deve manter os ovos em compartimento separado dos adultos e, após a fecundação, os ovos devem ser retirados e lavados, delicadamente, para evitar contaminações, para este fim pode ser utilizado o azul de metileno dissolvido na água $(0,00003 \%)$. Os embriões viáveis e não viáveis devem ser separados, utilizando-se apenas ovos viáveis para o desenvolvimento experimental.

\subsection{Importância do zebrafish nas pesquisas básicas e aplicadas}

Em 1822, o peixe zebra ou zebrafish foi descrito em um livro publicado pelo britânico Francis Hamilton, que entre os peixes encontrados no rio Ganges descreveu 10 exemplares do zebrafish (Danio rerio). O uso da espécie como modelo em estudos biomédicos e de embriologia foi registrado antes de 1930. Charles Creaser, em 1934, publicou um artigo sobre as facilidades de manusear e realizar estudos com a espécie. Em 1981, uma publicação do grupo de Streisinger (Universidade de Oregon) aprimorou o uso da espécie, produzindo embriões haploides a partir de ovos tratados com radiação ultravioleta e, assim, teve início o uso da espécie para pesquisas voltadas para a mutagênese, genética molecular, toxicologia, regeneração tecidual, entre outras áreas (GRUNWALD; EISEN, 2002). 
As áreas da neurologia, embriologia e farmacogenética apreciam este modelo animal por ser possível acompanhar as fases de desenvolvimento, ser um animal de fácil manipulação e fisiologia homóloga aos seres humanos (sistema nervoso central - SNC). O pequeno tamanho da espécie favorece alta estocagem, sendo possível manter 100.000 peixes como no maior criatório de peixes do Mundo (KALUEFF; STEWART; GERLAI, 2014). As instalações não necessitam infraestrutura complexa como nos biotérios de camundongos. O tempo de geração da espécie é de 3 meses e o espaço para criação requer apenas aquários de 8 a 12 L com 100 espécimes (DAMMSKI; MULLER, 2011). As fêmeas produzem cerca de 100 ovos ao dia (HOWE et al., 2013), os ovos são transparentes o que torna possível acompanhar e monitorar o desenvolvimento. Os embriões podem sobreviver alguns dias, após fertilização, sem alimentação externa e as drogas (testadas) se difundem facilmente através da pele e brânquias (LANTZ-MCPEAK et al., 2015).

Em pesquisas biomédicas, é possível estudar aplicações do modelo na neurologia, em estudos cardiovasculares, medicina regenerativa e alterações no comportamento. $\mathrm{Na}$ neurologia, estudo de biomarcadores para avaliar desordens no SNC, como: as doenças neurodegenerativas. O Alzheimer e o mal de Parkinson estão entre as doenças mais estudadas e o zebrafish tem sido modelo, pois o peixe possui um gene homólogo à proteína transmembrana ATP13A2 humana, que está associada com mal de Parkinson (LOPES DA FONSECA et al., 2013).

Estudos sobre o efeito tóxico de substâncias e contaminação do ambiente utiliza o zebrafish como biomarcador. $\mathrm{Na}$ Alemanha, os embriões foram introduzidos no teste ISO (ISO 15088:2007) para testes toxicológicos da água e, desde 2002, o teste em embriões de zebrafish para tratamento de águas residuais é obrigatório (BRAUNBECK, 2005.

Em trabalhos sobre medicina regenerativa, o zebrafish faz uma ponte entre o desenvolvimento celular e o biológico em função de seu rápido desenvolvimento. $O$ peixe vive até 5 anos em condições laboratoriais (GERHARD et al., 2002), facilitando estudos na área da regeneração e envolvendo o processo de envelhecimento (LAWRENCE, 2007). Como modelo em estudos para danos cardíacos, auxilia na busca por células e vias que podem recuperar pacientes cardíacos no processo de regeneração (GOESSLING; NORTH, 2014). Os fatores genéticos e ambientais, que influenciam na idade do cérebro, foram verificadas com o modelo e se concluiu que a expressão genética pode ser alterada com a idade e diferenciação sexual (ARSLAN-ERGUL; ADAMS, 2014).

\subsection{Biotério de manutenção}

$\mathrm{Na}$ instalação de um biotério se deve levar em conta fatores, como: o espaço, o valor do equipamento, o apoio técnico disponível na instituição. Deve-se verificar se a Universidade possui a documentação legal para instalação de biotério com licença para criação ou manutenção (com objetivo de manutenção de peixes em experimentação, por tempo superior a 12 horas).

Segundo a Resolução n ${ }^{\circ} 34$ / 2017 do Conselho Nacional de Controle de Experimentação Animal - CONCEA (BRASIL, 2017), em instalações nas quais a licença vigora para o uso laboratorial de manutenção da espécie, o peixe pode ser alocado em aquários com densidade de 5 peixes adultos/L, com aeração, biofiltro, renovação da água de $70 \%$ por dia, boa qualidade da água e regime de alimentação. Nos aquários sem biofiltro se deve respeitar a densidade de 1-2 animais por litro, manter aeração constante, renovação de $70 \%$ /dia, utilizar água de boa qualidade e bom regime de alimentação.

A água a ser utilizada na manutenção e conservação da espécie deve ser de fluxo contínuo e em laboratórios nos quais isso não seja possível e se deve preparar a água seguindo as seguintes proporções de produtos: solução de cloreto de cálcio (dissolver $11.76 \mathrm{~g} \mathrm{CaCl} 2.2 \mathrm{H} 20 \mathrm{em} 1 \mathrm{~L}$ de água deionizada); solução de sulfato de magnésio (dissolver 4.93 g MgS04.7H20 em água deionizada para 1L); solução de bicarbonato de sódio (dissolver 2.59 g NaHC03 em 1 L de água deionizada); solução de cloreto de potássio (dissolver $0.23 \mathrm{~g} \mathrm{KCl}$ em $1 \mathrm{~L}$ de água deionizada). $25 \mathrm{~mL}$ das soluções previamente preparadas devem ser misturadas e completadas para $1 \mathrm{~L}$ de água deionizada, a proporção de íons $\mathrm{Ca}$ : $\mathrm{Mg}$ deve ser de 4: 1 e íons Na: $\mathrm{K}$ de 10: 1 . A capacidade de ácido $\mathrm{K}_{\mathrm{S} 4.3}$ desta solução é 0,8 mmol / L (OECD, 1992). Antes de utilizar a água se deve manter a solução sob aeração por pelo menos 24 horas antes da experimentação.

Enriquecer o ambiente no qual os animais estão alocados favorece o bem-estar e tem influência no desenvolvimento do animal, devendo ser proporcional locais com cascalhos, plantas e locais nos quais o animal possa se abrigar. O zebrafish alocado em ambiente enriquecido com plantas artificiais evidenciou aumento da proliferação celular no telencéfalo (VON KROGH et al., 2010) e diminuiu comportamentos de tipo-ansiedade (COLLYMORE; TOLWANI; RASMUSSEN, 2015).

O tipo de alimentação oferecido para o zebrafish, em condições de manutenção, pode ser por alimentos industrializados, que dependerão de serem administrados de acordo com a idade do animal, o alevino requer ração com tamanho pequeno e que possa ser engolido inteiro, os animais juvenis maiores necessitam de uma demanda em quantidades maiores em relação aos alevinos. Além disso, pode ser oferecido para um zebrafish recém eclodido paramécios $(800 \mu \mathrm{m} \times 80 \mu \mathrm{m})$ e rotíferos. Os adultos de zebrafish podem receber a dieta em flocos, micropelets e os alimentos alternativos incluem larvas de mosca Drosophila e Artemia. Os alimentos devem ser adquiridos em pequenas quantidades para evitar contaminações, sempre levando em consideração as datas de fabricação e validade do produto (MATTHEWS; TREVARROW; MATTHEWS, 2002). 


\subsection{Parâmetros de cuidados e manutenção}

Em laboratório existe a necessidade de criar condições para garantir o bem-estar animal, pois o sucesso da experimentação, com resultados confiáveis depende dos cuidados aplicados para a manutenção dos peixes.

A manutenção do zebrafish em laboratório tenta recriar em cativeiro sistemas aquáticos de recirculação, que têm como objetivo garantir a qualidade da água e adequação à espécie. O controle rigoroso da qualidade da água é relevante, pois o animal está em contato direto com a água, através da pele e suas brânquias, tornando-se vulnerável a quaisquer alterações (COUTINHO, 2018; HARPER; LAURENCE, 2016).

A resolução do CONCEA n ${ }^{\circ} 34$ de 2017 visa garantir as condições adequadas de manutenção de peixes utilizados no ensino e na pesquisa para que os resultados possam ser confiáveis. Os princípios, que devem ser seguidos, desde o início do delineamento experimental até os cuidados na manutenção do animal, são conhecidos como os 3R das suas siglas em inglês (Replace, Reduce e Refine) (BRASIL, 2017).

\subsubsection{Parâmetros físico-químicos da água}

Os parâmetros de qualidade da água são essenciais para garantir o bem-estar e desenvolvimento do zebrafish. Os efeitos negativos impactam no sistema imunológico, fisiologia, estresse, resistência às patologias, comportamento e reprodução da espécie (HARPER; LAURENCE, 2016).

O zebrafish demonstra tolerância elevada para as mudanças de parâmetros físico-químicos da água, que estão relacionadas aos diferentes habitats na natureza, que são ocupados pela espécie. Entretanto, a manutenção da homeostasia do peixe fica comprometida em condições inapropriadas da água, verificando-se assim uma diminuição das posturas, no crescimento e influência sobre a sobrevivência (LAWERENCE, 2007).

\subsubsection{Temperatura}

O zebrafish é um animal pecilotérmico, não conseguindo regular sua temperatura corporal, dependendo assim da temperatura ambiente. O peixe tolera a variação de temperatura, podendo ser mantido por longo tempo as temperaturas entre 22 e $30{ }^{\circ} \mathrm{C}$ e podem sobreviver a temperaturas entre 18 e 32 ${ }^{\circ} \mathrm{C}$ (MATTHEWS; TREVARROW; MATTHEWS, 2002). Em condições experimentais de choque térmico, por exemplo, resiste a temperaturas acima de $30^{\circ} \mathrm{C}$.

A alteração de temperatura, em casos de falha no sistema de água, no qual o animal é mantido, é aceitável diminuir a taxa metabólica do animal expondo, gradualmente, a temperaturas de $22-23^{\circ} \mathrm{C}$. Entende-se que o aumento da temperatura, assim, aumenta o metabolismo do peixe, altera a respiração, aumentando o consumo de oxigênio da água e disponibilizando mais dióxido de carbono. As alterações na temperatura geram alterações no metabolismo, que aumentam a excreção de amônia (ALATORRE-JACOME et al., 2011).

\subsubsection{2 pH}

Amostragens de campo demonstram que o zebrafish habita águas, cujo $\mathrm{pH}$ se encontra e varia entre 5,9 e 8,1, entretanto, é recomendado que os laboratórios sejam mantidos em um valor constante entre 7 e 8 , pois favorecem assim melhor desenvolvimento e reprodução da espécie (ENGESZER et al., 2007; HARPER; LAWRENCE, 2016).

\subsubsection{Salinidade/Condutividade}

O zebrafish é um peixe de água doce e apresenta uma elevada tolerância à salinidade, podendo habitar em regiões com água salobra e em áreas com salinidade reduzida. Em ambiente de manutenção laboratorial se recomenda que a salinidade se mantenha entre 0,25 e $0,75 \mathrm{ppm}$. A condutividade da água deve se encontrar entre 300 e $1500 \mu \mathrm{S}$ (LAWRENCE, 2007; LAWRENCE; MASON, 2012).

\subsubsection{Oxigênio dissolvido}

O peixe utiliza o oxigênio dissolvido na água por absorção dos elementos químicos e trocas gasosas por capilares nas brânquias, sendo que a ausência de $\mathrm{O}_{2}$ pode comprometer a respiração, crescimento, alimentação e sobrevivência do animal (ALATORRE-JACOMEM et al., 2011). A concentração mínima recomendada em animais, sob manutenção de laboratório, é de $7,8 \mathrm{mg} / \mathrm{L}$ para garantir o bem-estar e qualidade de desenvolvimento (HAPER; LAWRENCE, 2016).

As práticas que utilizam os animais em laboratório voltados para experimentação devem seguir com rigidez os preceitos éticos e protocolos de manutenção do zebrafish. Dessa forma, são fundamentais os métodos e protocolos para manutenção, desenvolvimento e reprodução da espécie.

\section{Conclusão}

A revisão de literatura oferece a compilação de possíveis recomendações para manter espécimes de zebrafish em laboratórios de ensino e pesquisa de instituições, que possuem apenas autorização para manutenção. Os animais que são mantidos em condições laboratoriais, respeitando o princípio dos 3Rs e mantendo os parâmetros físicos e químicos regulados, proporcionam melhores resultados experimentais, de desenvolvimento e de reprodução. Portanto, é necessário levar em consideração os protocolos de manutenção propostos por organizações reguladoras como foi sintetizado no trabalho.

\section{Referências}

ALATORRE-JACOME, O. et al. Aquaculture Water Quality for Small-Scale Producers. In: Aquaculture and the Environment - A Shared Destiny. Querétaro: InTech. 2011. p. 125-140.

ALESCO. Rack Hydrus para Zebrafish. Disponível em: <https:// www.alesco.com.br/rack-para-zebrafish-hydrus $>$. Acesso em: 28 jun. 2020.

ARSLAN-ERGUL, A.; ADAMS, M.M. Gene expression changes in aging Zebrafish (Danio rerio) brains are sexually dimorphic. 
BMC Neurosci., v.15, p.29, 2014.

BRASIL. Resolução CONCEA $n^{\circ}$ 34, de 27 de julho de 2017. Disponível em: <http://antigo.mctic.gov.br/mctic/export/ sites/institucional/institucional/concea/arquivos/legislacao/ resolucoes_normativas/Resolucao-Normativa-CONCEA-n-34de-27.07.2017-D.O.U.-de-31.07.2017-Secao-I-Pag.-218-Anexo. pdf. $>$. Acesso em: 19 jun. 2020.

BRAUNBECK, T. et al. Towards an alternative for the acute fish LC50 test in chemical assessment: The fish embryo toxicity test goes multi-species: an update. Altex, v.22, n.2, p.87-102, 2005.

COLLYMORE, C.; TOLWANI, R.J.; RASMUSSEN, S. The behavioral effects of single housing and environmental enrichment on adult zebrafish (Danio rerio). J. Am. Assoc. Lab. Anim. Sci., v.54, n.3, p.280-285, 2015.

COUTINHO, C. I. N. Manutenção e reprodução em peixe-zebra (Danio rerio): relatório final de estágio no Biotério de Organismos Aquáticos do CIIMAR. Porto: Universidade do Porto, 2018.

D'ANGELO, L. et al. Anatomical features for the adequate choice of experimental animal models in biomedicine: I. Fish. Ann. Anat., v.205, p.75-84, 2016. doi: 10.1016/j.aanat.2016.02.001.

DAMMSKI, A.P. et al. Zebrafish: manual de criação em biotério. Curitiba: Universidade Federal do Paraná, 2011.

ENGESZER, R. E. et al. Zebrafish in the wild: A review of natural history and new notes from the field. Zebrafish, v.4, n.1, p.21-40, 2007. doi: 10.1089/zeb.2006.9997.

GEISLER, R. et al. Maintenance of Zebrafish Lines at the European Zebrafish Resource Center. Zebrafish, v.13, p.19-23, 2016. doi: 10.1089/zeb.2015.1205.

GERHARD, G. S. et al. Life spans and senescent phenotypes in two strains of Zebrafish (Danio rerio). Exp. Gerontol., v.37, n.8-9, p.1055-1068, 2002. doi: 10.1016/S0531-5565(02)00088-8.

GOESSLING, W.; NORTH, T. E. Repairing quite swimmingly: Advances in regenerative medicine using zebrafish. Dis. Model. Mec., v.7, n.7, p.769-776, 2014.

GOODWIN, N. et al. Standardized welfare terms for the zebrafish community. Zebrafish, v.13, p.164-168, 2016a.

GOODWIN, N. et al. Evaluating and optimizing fish health and welfare during experimental procedures. Zebrafish, v.13, p.127131, 2016b.

GRUNWALD, D.J.; EISEN, J.S. Headwaters of the zebrafish: emergence of a new model vertebrate, Nat. Rev. Genet., v.3, n.9, p.717-724, 2002. doi: 10.1038/nrg892.
HARPER, C.; LAWRENCE, C. The Laboratory Zebrafish. Boca Raton: CRCpress, 2016.

HOWE, K. et al. The zebrafish reference genome sequence and its relationship to the human genome. Nature, v.496, n.7446, p.498-503, 2013. doi: 10.1038/nature12111.

KALUEFF, A.V.; STEWART, A.M.; GERLAI, R. Zebrafish as an emerging model for studying complex brain disorders. Trends Pharmacol. Sci, v.35, n.2, p.63-75, 2014.

LANTZ-MCPEAK, S. et al. Developmental toxicity assay using high content screening of zebrafish embryos. J. Appl. Toxicol., v.35, n.3, p.261-272, 2015.

LAWRENCE, C. The husbrandry of zebrafish (Danio rerio): a review. Aquaculture, v.269, n.1, p.1-20, 2007. doi: 10.1016/j. aquaculture.2007.04.077.

LAWRENCE, C.; EISEN, J. S.; VARGA, Z. M. Husbandry and health program survey synopsis. Zebrafish, v.13, n.S1, p.S-5-S-7, 2016.

LAWRENCE, C.; MASON, T. Zebrafish housing systems: a review of basic operating principles and considerations for design and functionality. ILAR J., v.53, n.2, p.179-191, 2012. doi: 10.1093/ilar.53.2.179.

LIDSTER, K. et al. International survey on the use and welfare of zebrafish Danio rerio in research. J. Fish Biol., v. 90, p. 18911905, 2017.

LOPES DA FONSECA, T. et al. The zebrafish homologue of Parkinson's disease ATP13A2 is essential for embryonic survival. Brain Res. Bull., v.90, p.118-126, 2013. doi: 10.1016/j. brainresbull.2012.09.017.

MATTHEWS, M.; TREVARROW, B.; MATTHEWS, J. A virtual tour of the Guide for zebrafish users. Lab Ani., v.31, n. 3, p.3440, 2002.

OECD. Fish, acute toxicity test. Guideline for the testing of chemicals, 1992.

VON KROGH, K. et al. Forebrain cell proliferation, behavior, and physiology of zebrafish, Danio rerio, kept in enriched or barren environments. Physiol. Behav., v.101, n.1, p.32-39, 2010. doi: 10.1016/j.physbeh.2010.04.003. 